\title{
Design and Implementation of Aquarium Remote Automation Monitoring and Control System
}

\author{
Yinchi $\mathrm{Ma}^{1,2, *}$ and Wen Ding ${ }^{1,2}$ \\ ${ }^{1}$ Beijing Fisheries Research Institute, Beijing, 100068, China \\ ${ }^{2}$ National Engineering Research Center of Freshwater Fisheries, Beijing, 100068, China
}

\begin{abstract}
In recent years, with the rapid development of ornamental fisheries and recreational fisheries, different varieties of ornamental fish have come into many office business palaces and people's house. The Aquarium and many other aquarium equipment are developing from functionality to intelligentize. Through the industry research, a set of remote automation monitoring and control equipment for the aquarium will have important significance and development prospect. The system stores and transmits the video of the aquarium gathered by camera through the IMagineWorld center platform software. The Internet user accesses the center platform to monitor the aquarium by IE or mobile terminal software. The user can get the temperature data of the aquarium through RS485 transparent protocol. And the user can also control the oxygen adding, warmer and filtering equipment remotely by the $\mathrm{I} / \mathrm{O}$ alarm output of the video server. Through the user testing, the system plays a good effect and has wide application and popularization value.
\end{abstract}

Keywords: aquarium, remote, automation, monitoring and control.

\section{Introduction}

At present, there is a wide variety of aquarium in the market. And some multifunction aquariums such as controlling water temperature, increasing oxygen, and filtering, feeding, and other functions have appeared. But most functions need to be operated manually in site. With the progress of modern electronic information and automation technology, in some fields such as intelligent manufacturing, precision agriculture, and household, have been able to realize the remote video monitoring, environmental parameters monitoring, equipment condition monitoring and controlling [1]. These technologies have had some simple application on the aquarium equipment, but the multi-functional integration application is still a blank in this area. Tu used the on-line video to monitor the oil contamination level [2]. Peng, Yao, Zhang and Yu monitored and controlled the aquarium equipments based single-chip computer technology, but

\footnotetext{
* Ma Yinchi (1982 -), Beijing Fisheries Research Institute, Engineer, Master, graduated from Beijing Normal University, State Key Laboratory of Remote Sensing Science, mainly engaged in research of agriculture remote sensing and fisheries information technology.
} 
not realized the remote control and the on-line remote video monitoring [3] - [6]. Ge realized the remote control of the aquarium equipments based RS-485 bus. But the dynamic IP problem was not solved [7]. Yang realized to control the oxygen adding equipment of the fish pond based GSM and WIFI technology [8]. Through analysis and study of the most aquarium controlling equipment application status at present, people can't stand work of the aquarium, and also cannot monitor or control the electrical equipment of the aquarium in real-time. These problems cause a lot of trouble for daily maintenance, and even cause some economic losses [9]. In this article, we propose a system solution method that can realize remote automation monitoring and controlling of the aquarium. By applying IMagineWorld center platform, RS485 protocol software and I/O alarm output control technology, the system can realize to view video of the aquarium, to monitor temperature of the water, to add oxygen, and to warm or filter the water based through the Internet. At the same time, The dynamic IP problem is also solved which makes the system convenient for simple household application..The design and implementation of this system will have an important innovation meaning for the design and manufacture area of the aquarium.

\section{System Composition}

The System is composed of the monitoring module, the control module, the video server, the center server platform and the client.

The monitoring module monitors the real-time video and the water temperature of the aquarium.

The control module is designed to control the light equipment, the heating equipment, the oxygen adding equipment and the filtering equipment.

The video server is the core part of the system, and we have improved and expanded its function. The I/O alarm output function of the video server is encapsulated into the system I/O control unit. The Unit receives all kinds of orders sent from the center server platform, and controls the equipment belonging to the control module in the aquarium. At the same, the unit encodes the data from the temperature sensor and uploads the data to the center sever platform through the RS485 transparent protocol. The real-time video data of the aquarium gathered by CCD sensor is compressed and coded, and then is also uploaded to the center server platform automatically. These functions are encapsulated into the data service unit.

The IMagineWorld management software is installed on the center server platform. The software can deal with the temperature and video data sent from the video server, and put forward these data to the request client. At the same time, all the monitoring and controlling orders by user are also sent to the control unit of the remote video server through the platform.

The system client is divided to two kinds. For normal PC users, we design an internet server based on the ASP technology on the center server platform. The users can visit the system and use all functions through internet by IE explorer. For other mobile users, 
we design client software running on the Android mobile operation system platform. The users can use the system through GPRS network or WIFI network by the client. Considering the safety and privacy of users, the system has a strict user authentication mechanism. All the monitoring and controlling operations from IE or the client must base on the legitimate login.

The structure design of the system is shown as follow (Fig.1).

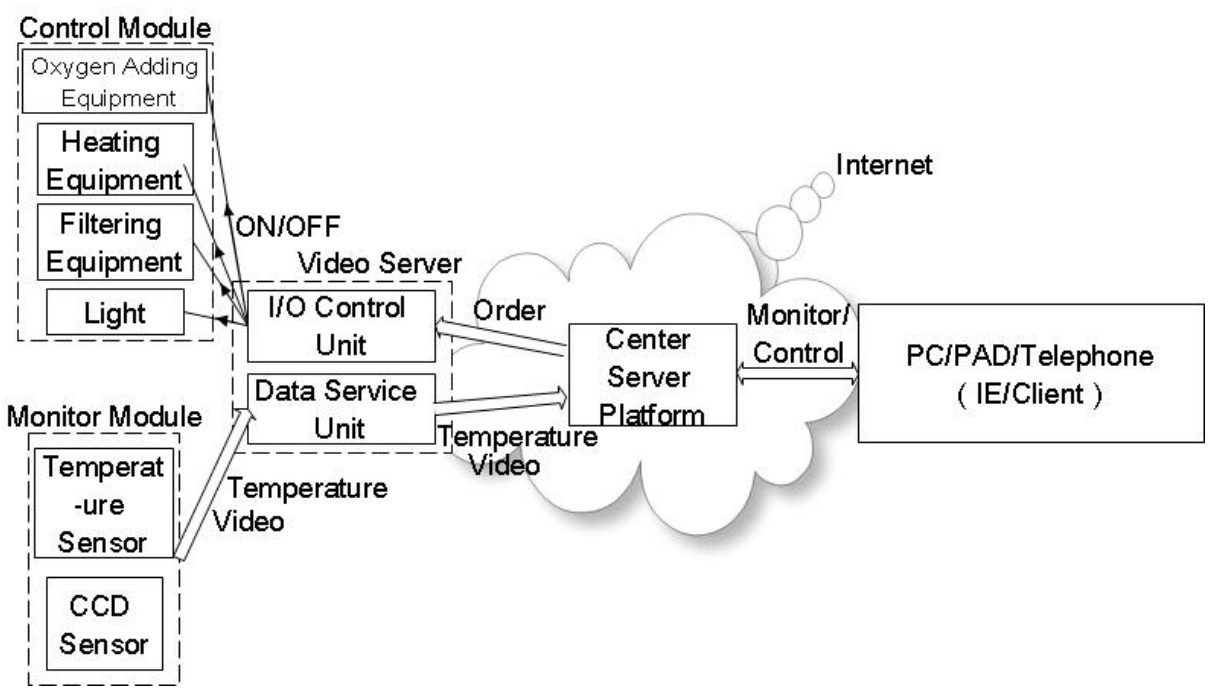

Fig. 1. System Structure Design

\subsection{Hardware}

The system hardware consists of the control module, the monitoring module, the video server and the web server.

Based on the current popular aquarium equipment, the control module consists of the light, the heating equipment, the filtering equipment and the oxygen adding equipment. These equipments are controlled by an independent circuit. There are control buttons on the control panel of the aquarium. And every control circuit is connected to the I/O control unit of the video server. This design realized the local control and the remote control at the same time.

At present, the monitoring module is composed by the water temperature sensor and the real-time video CCD sensor. The tiny custom water temperature sensor is applicable to the household aquarium. The DS18B20 component uses the waterproof packaging technology. The sensor is connected to the video server by leading wire. Its testing range is between $-55^{\circ} \mathrm{C} \sim 125^{\circ} \mathrm{C}$. And the accuracy of the sensor is $\pm 0.5^{\circ} \mathrm{C}$ working 
under the $-10^{\circ} \mathrm{C} \sim 85^{\circ} \mathrm{C}$ condition. The Temperature data is conversed to RS485 signal through the video server. And then the video server sends these data to the remote center service platform by internet or GPRS network. User can set up the time interval of the water temperature refreshment in the system, and the temperature data can be refreshed on the client or IE automatically. The video acquisition device encapsulates a color CCD sensor with 420 line horizontal resolution. It uses PAL or NTSC signal mode. The camera has wide-angle function and can eliminate spherical deformation of the video. The camera base uses the sucker structure, and the reinforced sucker can fix the camera on the outer wall of the aquarium hardly. User can change the location and angle of the camera due to requirement. The camera is connected to the video server by $1.0 \mathrm{Vp}-\mathrm{p} / 75 \Omega / \mathrm{BNC}$ interface.

In recent years, the video server has been widely used in remote monitoring and security area. It is a kind of embedded device to compress and deal with the audio and the video data. It is composed of AV compression and encoder device, input and output channel, network interface, AV interface, RS485/RS232 serial interface, protocol interface and software interface, etc.

This system is equipped with a high quality video server with high quality SOC computing chip which is stable and reliable, and has low power consumption. The video server uses the H.264 video coding technology with a high compression ratio and high image quality. The video server supports NTSC or PAL video formats and its image resolution can reach $704 * 576$ pixels, and the video compression rate is $32 \mathrm{kbps} \sim 4 \mathrm{Mbps}$, the most high frame rate can be up to 30 frames per second. The video server can support 4 alarm input signal and 4 alarm output switch signal. It has the RJ45 adaptive 10M/100M Ethernet port, and supports transparent RS485 reading and writing protocol. The video server has a dynamic DNS client inside, and can be configured as the secondary domain name. This makes it particular convenient to the family dynamic IP users. The video server uses the DC12V/2A ballast for power supply.

We design an I/O power controller for the video server, so as to control the switch of the aquarium equipment in remote way. The main structure components are composed of a transparent cover, an upper cover body, a fixing rail and a terminal block, etc. All the built-in electric switch components adopt with $9 \mathrm{~mm}$ module electric equipment, and are fixed on the fixing rail. The Grounding is firm and reliable, and can be convenient to use. The controller is equipped with 4 groups of $10 \mathrm{~A}$ socket, $12 \mathrm{~V} / 5 \mathrm{~A}$ switch power and $412 \mathrm{~V}$ relays. The 4 groups of I/O switch control the 4 relays, and the $12 \mathrm{~V}$ switch power supplies power to the RS485 water temperature sensor and the 4 relays. The inside circuit structure of the $\mathrm{I} / \mathrm{O}$ power controller is shown as follow (Fig.2).

\subsection{Software}

The network system service is setup on the center server platform, and the IMagineWorld software is also setup on it. IMagineWorld is the core software of the center server platform, and is responsible for storing and forwarding of the real-time 


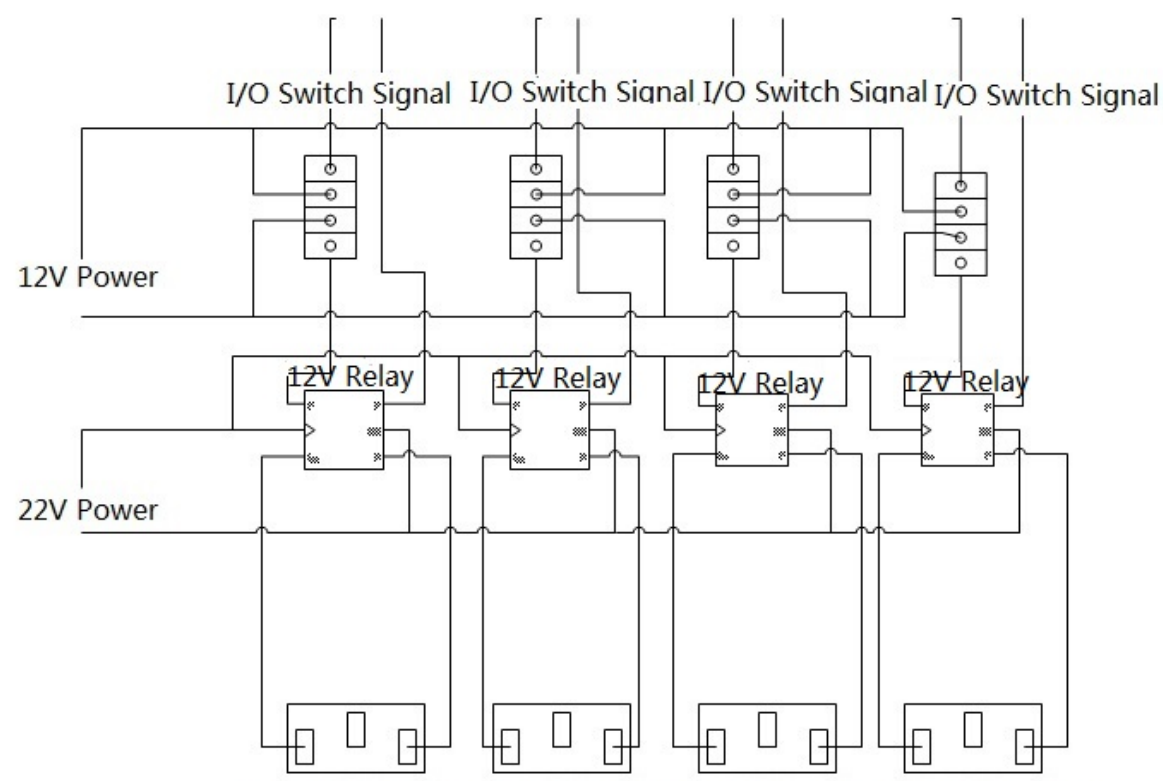

220V10A Socket 220V10A Socket220V10A Socket220V10A Socket

Fig. 2. Inside Circuit Structure of I/O Power Controller

video. After the system administrator checks the count of the user who owns the system equipment, he will add the device ID into the system service list. The device ID is the only identifier of the system equipment. Only the system equipment whose device ID recorded in the system service list can upload the real-time video to the center server platform, then store and forward the video to the remote client through the center server platform.

The network users can visit the system through internet by IE, and they can monitor and control the aquarium by the IE widgets. We setup a set of rules for the user management. User must register a count on the network system first, and then the system administrator will check it. The center server platform can bind one or more sets of the system equipment for every user, and the user can switch among these equipments through the client or IE interface.

Aiming at the development of smart mobile terminals, for the convenience to monitor and control the remote aquarium anytime and anywhere, we develop a client for this system based on Android smart mobile operating system platform. For the legal users who have registered successfully, they can download and installed the client on their phone or pad running the Android system. And then they can login and use the system freely. The mobile client not only provides all the functions of the web system, but also provides image snapshot and video record function. The center server compresses the transfer data, and optimizes the network resource occupancy and data traffic. So users can operate the system through GPRS or WIFI network smoothly. 


\section{Conclusions}

In this paper, we make detail design and implementation for remote automation monitoring and controlling of the aquarium. The structure of the system is simple and the performance is reliable. After strict system test, the system has obtained good effect. The aquarium water temperature data can be sent to the user client in real-time through the internet. Users can remote control the heating device due to the temperature data initiatively. And at the same time, users can remote control the oxygen adding device and the filtering device due to the video data from the CCD sensor initiatively.

Monitoring and controlling the aquarium in real-time can ensure the breeding security and reduce the breeding risk and loss. The application of this system can eliminate the influence of human factors during the breeding process, and increase efficiency significantly. For home and commercial places aquarium users, the beautiful and lightweight hardware structure design is important. And the software interface is also required to be friendly and operable. We will optimize and improve the system for these requirements. Industrial hardware structure design of the system can make it product and apply at scale. And the system will have a more practical usage. To expand the control circuit and do modular design will allow users to easily customize their own remote monitoring and controlling module in this system. And to expand the operating system platform of the mobile client will strengthen the portability of the system software. At the same time, we also design a particular system that can be used in large breeding sink to expand the system application.

\section{References}

1. Zhou, G., Zhou, J., Miao, Y., et al.: Development and application on GSM-based monitoring system for digital agriculture. Transactions of the CSAE 21(6), 87-91 (2005) (in Chinese with English abstract)

2. Tu, Q., Zuo, H.: Study on a New Technique of On-line Monitoring of Oil Contamination Level Using Computer Vision Technology. International Journal of Plant Engineering and Management (02), 103-109 (2004)

3. Peng, G., Deng, H., Liang, Z.: The Design of Automatic-Control Instrument for the Aquarium. Guangdong Automation \& Information Engineering Z1, 44-46 (1998) (in Chinese with English abstract)

4. Yao, L., Yang, Y.: The design of aquatic animal case/s auto adding oxygen system. Journal of Shenyang Institute of Aeronautical Engineering 25(1), 64-66 (2008) (in Chinese with English abstract)

5. Zhang, J.: Making the oxygen pump control switch of aquarium with single chip microcomputer. Electronics World (10), 28-29 (2004) (Chinese reverse to English)

6. Yu, H., Shan, H., Jiang, L.: Design of the automation oxygen controller based single chip microcomputer. Fishery Modernization (6), 42-44 (2006) (Chinese reverse to English) 
7. Ge, H.: The Realization of the Remote Communication between PC and the Controller in Aquarium Based on RS-485 Bus. Journal of Shazhou Professional Institute of Technology 12(3), 8-11 (2009) (in Chinese with English abstract)

8. Yang, S., Qi, J., Li, Y., et al.: Wireless system for monitoring and control of dissolved oxygen in fish pond water. Fishery Modernization 37(6), 11-14 (2010) (in Chinese with English abstract)

9. Zheng, W.: Technology of Breeding Seawater Fancy Fishes in Household Aquarium. Fisheries Economy Research (1), 45-48 (2009) 